\title{
NUMERICAL SIMULATIONS OF THZ PULSE GENERATION WITH TWO-COLOR LASER PULSES IN THE 2.15-15.15 $\mu \mathrm{m}$ SPECTRAL RANGE
}

\author{
Roland Flender ${ }^{1}$, Adam Borzsonyi ${ }^{1,2}$, Viktor Chikan ${ }^{1,3}$
}

\author{
${ }^{1}$ ELI-ALPS, ELI-HU Non-Profit Ltd., Wolfgang Sandner utca 3., Szeged, H-6728, Hungary \\ ${ }^{2}$ Dept. of Optics and Quantum Electronics, Univ. of Szeged, Dóm tér 9., H-6720 Szeged, Hungary \\ ${ }^{3}$ Dept. of Chemistry, Kansas State University, 213 CBC Building, Manhattan, KS 66506-0401, USA.
}

DOI: https://doi.org/10.14232/kvantumelektronika.9.9

\section{Introduction}

The terahertz (THz) pulse generation from two-color laser pulse ionized gases is an actively researched topic because of its simplicity and practical use in renewable secondary sources for ultrafast technology. In this method, the two-color laser pulses, the fundamental beam and its second harmonic are focused in ambient air or in different gases (see Fig. 1.). After the tunnel ionization, the free electrons accelerate in the external electric field of the laser pulses. When the relative phase between the fundamental and its second harmonic is $\pm \pi / 2 \mathrm{rad}$, the acceleration becomes asymmetric and a significant net electron current develops inside the plasma [1,2]. This phase-sensitive electron current is the source of the $\mathrm{THz}$ pulse.

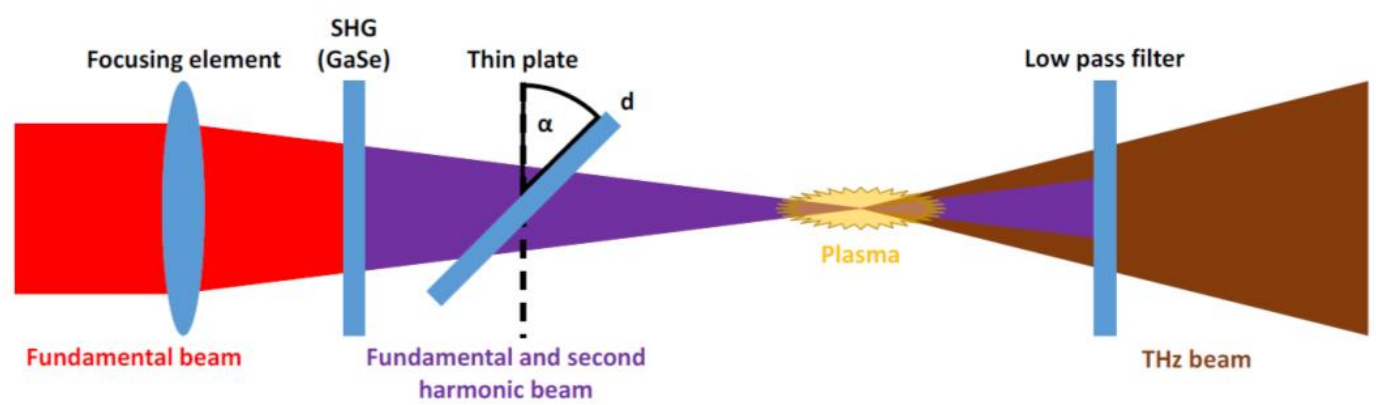

Fig.1. Schematic diagram of the THz pulse generation in air by two-color laser pulses

Several groups have demonstrated the efficiency of $\mathrm{THz}$ pulse generation increase with the central wavelength of the fundamental laser pulse [3-8]. A key reason behind this observation is the small temporal walk-off between the mid-infrared (MIR) two-color laser pulses compared to the shorter wavelengths $[6,7]$. On the other hand, the smaller temporal walk-off makes it more difficult to control the relative phase of the two-color laser pulses.

\section{Results}

The laser pulse parameters for the numerical simulations are based on the MIR laser system [9, 10] located at ELI-ALPS user facility [11]. The central wavelength of this laser system is $3.2 \mu \mathrm{m}$ and the spectral bandwidth supports $45 \mathrm{fs}$ pulse duration, the pulse energy at the output window is $140 \mu \mathrm{J}$. The central wavelength of the laser system is tunable between $2.5 \mu \mathrm{m}$ and $4 \mu \mathrm{m}$, but in this simulation we expanded the spectral range from $2.15 \mu \mathrm{m}$ up to $15.15 \mu \mathrm{m}$, while keeping the pulse duration, expressed in optical cycles and the peak power at a constant value. The investigated spectral region is defined by the gallium selenide (GaSe) crystal properties, which is used for the second harmonic generation (SHG) process. 
In this research, we examined numerically the $\mathrm{THz}$ pulse generation in the spectral range between $2.15 \mu \mathrm{m}$ and $15.15 \mu \mathrm{m}$. The $\mathrm{THz}$ pulse spectrum and peak of its electric field intensity in the function of the central wavelength of the fundamental pulse is shown in Fig. 1., in case of various commercially available low-pass $\mathrm{THz}$ filters [12]. Initially, the $\mathrm{THz}$ pulse electric field increase continuously with the central wavelength of the fundamental laser pulse up to $14.30 \mu \mathrm{m}$. The generated $\mathrm{THz}$ pulse electric field is hundred times stronger compared to the Ti:Sapphire lasers, which is the most commonly used lasers for this type of THz pulse generation. The mechanism behind this is that the amplitude of the THz electric field is proportional to the first derivative of the transverse electron current that is proportional to the velocity of the electrons and the density of the electrons. The velocity of the electrons is proportionate to the combined electric field and the asymmetry of this field. The asymmetry of the electric field changes significantly in the function of the central wavelength of the fundamental pulse. The asymmetry of the electric field yields faster electrons as a function of the central wavelength of the fundamental laser pulse. The velocity of the electrons increases the transverse electron current needed for the more intense THz pulse intensity $[1,2]$.

(a)

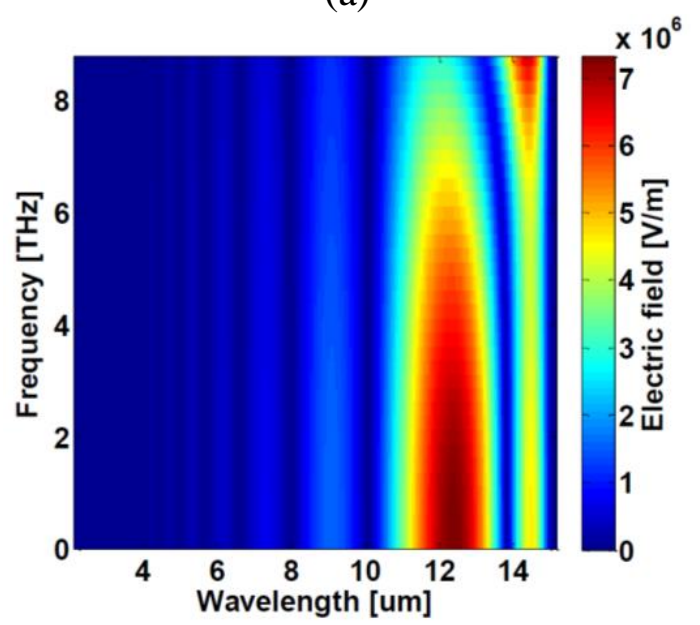

(b)

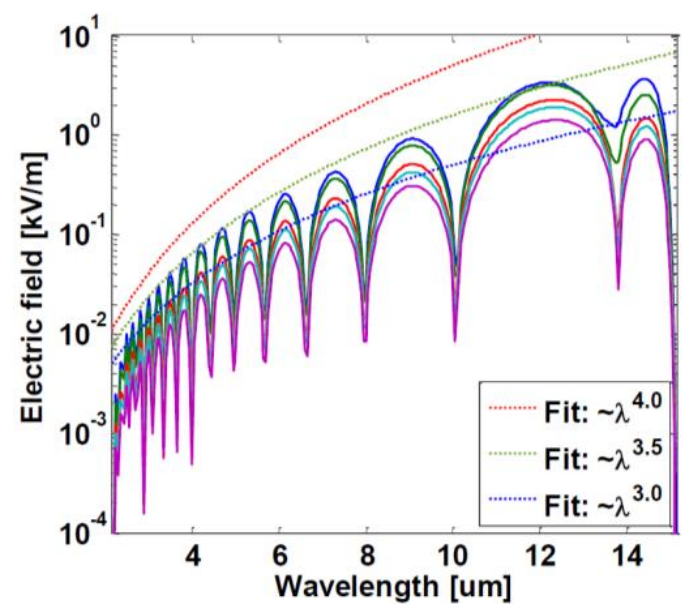

Fig.2. Terahertz pulse spectrum (a) and peak electric field (b) as a function of the fundamental pulse central wavelength in the case of different terahertz low-pass filters.

In addition to the above results, we have explored the use of barium-fluoride $\left(\mathrm{BaF}_{2}\right)$, calcium-fluoride $\left(\mathrm{CaF}_{2}\right)$ and lithium-fluoride $(\mathrm{LiF})$ crystals to control the relative phase between the fundamental pulse and its second harmonic. The first two materials are alkaline earth halides [13] and the latter one is an alkali halide [14]. The relative phase control ability of these plates are shown in Fig.3.a-c.

(a) $\mathrm{BaF}_{2}$

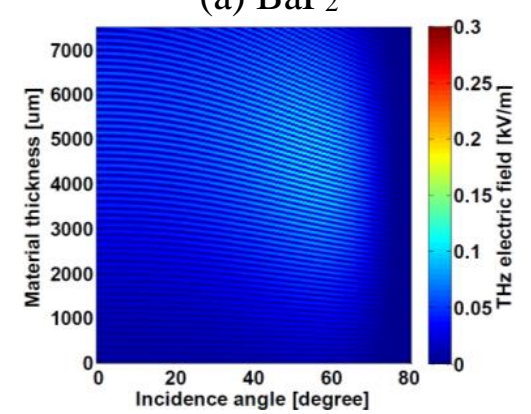

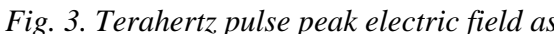

(b) $\mathrm{CaF}_{2}$

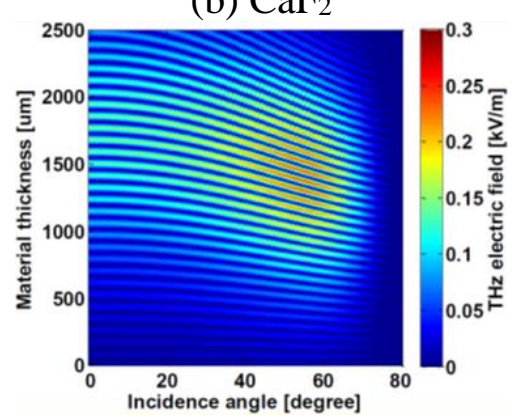

(c) $\mathrm{LiF}$

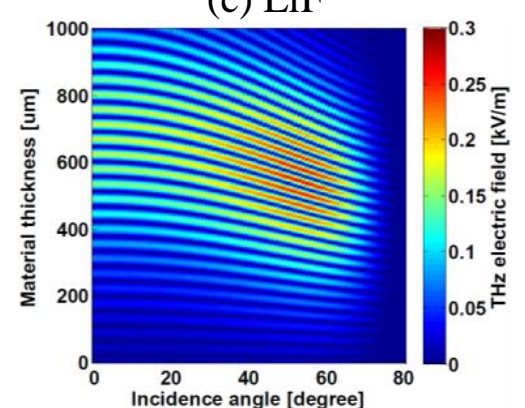

(b) $\mathrm{CaF}_{2}$ and (c) LiF, respectively.
(c) 
Let us discuss LiF (in Fig. 3.c) in particular. As one can see the $\mathrm{LiF}$ is capable to control the relative phase of the two-color laser pulse and initially capable to increase the generated THz pulse intensity. The phase velocity difference between the $3.2 \mu \mathrm{m}$ and $1.6 \mu \mathrm{m}$ in $\mathrm{LiF}$ is few thousand times more than in ambient air allowing the reduction of size of optical setup needed to control the phase. The optimal thickness of the LiF plate is $540 \mu \mathrm{m}$ and the optimal incidence angle is $55.0^{\circ}$ degree. Based on our simulations, all the three fluorides are capable to control the relative phase of the two-color laser pulses with various efficiencies.

\section{Summary}

In summary, we have examined the THz pulse generation in the MIR spectral region. We have found that in the spectral range from $2.15 \mu \mathrm{m}$ up to $15.15 \mu \mathrm{m}$, the generated $\mathrm{THz}$ pulse intensity continuously increases with the fundamental pulse central wavelength up to $12.30 \mu \mathrm{m}$, where it reaches its maximum value. We have examined the control of the relative phase with co-propagation of the pulses through a thin plate. We have found that several fluorides are not just capable to control the relative phase of the two-color pulses, but also capable to improve the temporal overlap, whereupon they are capable to improve the $\mathrm{THz}$ pulse generation efficiency. We have found that the best candidate for good phase control is lithium fluoride.

\section{Acknowledgement}

The ELI-ALPS project (GINOP-2.3.6-15-2015-00001) is supported by the European Union and cofinanced by the European Regional Development Fund. The project has been supported by the European Union, co-financed by the European Social Fund. EFOP-3.6.2-16-2017-00005-"Ultrafast physical processes in atoms, molecules, nanostructures and biological systems“.

\section{References}

[1] Y. Watanabe et al. Opt. Comm. 234, 125-129 (2004)

https://doi.org/10.1016/j.optcom.2004.02.032

[2] J. Dai et al. App. Phys. Lett. 94(2), 021117 (2009)

https://doi.org/10.1063/1.3068501

[3] J. Liu et al. Nat. Phot. 4(9), 627-631 (2010)

https://doi.org/10.1038/nphoton.2010.165

[4] M. Clerici et al. Phys. Rev. Lett. 110(25) 253901 (2013)

https://doi.org/10.1103/PhysRevLett.110.253901

[5] A. Nguyen et al. Opt. Exp. 25(5) 4720-4740 (2017)

https://doi.org/10.1364/OE.25.004720

[6] A. Nguyen et al. Phys. Rev. A 97(6) 063839 (2018)

https://doi.org/10.1103/PhysRevA.97.063839

[7] V. Y. Fedorov et al. Phys. Rev. A 97(6) 063842 (2018)

https://doi.org/10.1103/PhysRevA.97.063842

[8] A. Nguyen et al. Opt. Lett. 44(6) 1488-1491 (2019)

https://doi.org/10.1364/OL.44.001488

[9] N. Thiré et al. Opt. Exp. 25(2) 1505-1514 (2017)

https://doi.org/10.1364/OE.25.001505 
[10] N. Thiré et al. Opt. Exp. 26(21) 26907-26915 (2018)

https://doi.org/10.1364/OE.26.026907

[11] http://www.eli-alps.hu

[12] http://www.tydexoptics.com/products/thz_optics/thz_low_pass_filter

[13] H. H. Li, J. Phys. Chem. Ref. Data 9(1), 161-290 (1980)

https://doi.org/10.1063/1.555616

[14] H. H. Li, J. Phys. Chem. Ref. Data 5(2), 329-528 (1976)

https://doi.org/10.1063/1.555536 